\title{
Systematic Review of the Impact of Transcranial Direct Current Stimulation on the Neuromechanical Management of Foot and Ankle Physical Performance in Healthy Adults
}

\author{
Songlin Xiao ${ }^{1}$, Baofeng Wang ${ }^{1}$, Xini Zhang ${ }^{1}$, Junhong Zhou ${ }^{2,3 *}$ and Weijie Fu ${ }^{1,4 *}$ \\ ${ }^{1}$ School of Kinesiology, Shanghai University of Sport, Shanghai, China, ${ }^{2}$ The Hinda and Arthur Marcus Institute for Aging \\ Research, Hebrew SeniorLife, Boston, MA, United States, ${ }^{3}$ Harvard Medical School, Boston, MA, United States, ${ }^{4}$ Key \\ Laboratory of Exercise and Health Sciences of Ministry of Education, Shanghai University of Sport, Shanghai, China
}

OPEN ACCESS

Edited by:

Borja Sañudo,

Sevilla University, Spain

Reviewed by:

Maryam Zoghi,

La Trobe University, Australia

Chi-Wen Lung,

Asia University, Taiwan

*Correspondence:

Weijie Fu

fuweijie@sus.edu.cn

Junhong Zhou

junhongzhou@hsl.harvard.edu

Specialty section: This article was submitted to

Biomechanics,

a section of the journal Frontiers in Bioengineering and

Biotechnology

Received: 27 July 2020 Accepted: 09 October 2020 Published: 30 October 2020

Citation:

Xiao S, Wang B, Zhang $X$, Zhou J and Fu W (2020) Systematic Review of the Impact of Transcranial Direct Current Stimulation on the Neuromechanical Management of Foot and Ankle Physical Performance in Healthy Adults.

Front. Bioeng. Biotechnol. 8:587680. doi: 10.3389/fbioe.2020.587680
Objective: This study aims to review existing literature regarding the effects of transcranial direct current stimulation (tDCS) on the physical performances of the foot and ankle of healthy adults and discuss the underlying neurophysiological mechanism through which cortical activities influence the neuromechanical management of the physical performances of the foot and ankle.

Methods: This systematic review has followed the recommendations of the Preferred Reporting Items for Systematic reviews and Meta-Analyses. A systematic search was performed on PubMed, EBSCO, and Web of Science. Studies were included according to the Participants, Intervention, Comparison, Outcomes, and Setting inclusion strategy. The risk of bias was assessed through the Cochrane Collaboration tool, and the quality of each study was evaluated through the Physiotherapy Evidence Database (PEDro) scale.

Results: The electronic search resulted in 145 studies. Only eight studies were included after screening. The studies performed well in terms of allocation, blinding effectiveness, and selective reporting. Besides, the PEDro scores of all the studies were over six, which indicated that the included studies have high quality. Seven studies reported that tDCS induced remarkable improvements in the physical performances of the foot and ankle, including foot sole vibratory and tactile threshold, toe pinch force, ankle choice reaction time, accuracy index of ankle tracking, and ankle range of motion, compared with sham.

Conclusion: The results in these studies demonstrate that tDCS is promising to help improve the physical performances of the foot and ankle. The possible underlying mechanisms are that tDCS can ultimately influence the neural circuitry responsible for the neuromechanical regulation of the foot and ankle and then improve their physical performances. However, the number of studies included was limited and their sample sizes were small; therefore, more researches are highly needed to confirm the findings of the current studies and explore the underlying neuromechanical effects of tDCS.

Keywords: transcranial direct current stimulation, foot, ankle, physical performance, neural circuitry, neuromechanical management 


\section{INTRODUCTION}

The functionality and physical performances of foot and ankle, including muscular strength, somatosensory function, and endurance, play a key role in locomotor control when standing, walking, jumping, and endurance running in everyday activities (Rodgers, 1988; Aagaard, 2018). In addition to the peripheral nervous system, the cortical functional networks of the brain have been linked to the formation and regulation of the physical performances of the foot and ankle (Noakes, 2011; Foerster et al., 2018a). A decrease in the excitability of cortical regions is associated with diminished biomechanical management of physical performance and increases the risk of injuries (e.g., chronic ankle inability) (Needle et al., 2017). Therefore, strategies designed to facilitate the neural circuitry of the brain have a great potential of improving the functional and physical performances of the foot and ankle.

One promising approach is transcranial direct current stimulation (tDCS). TDCS non-invasively modulates the excitability of brain regions by delivering low-amplitude current flow between two or more electrodes placed on the scalp (Nitsche and Paulus, 2000, 2001; Reardon, 2016). Anodal tDCS can increase cortical excitation through the tonic depolarization of the membrane resting potential, and cathodal tDCS may decrease cortical excitation by the hyperpolarization of the membrane resting potential (Stagg and Nitsche, 2011; Rahman et al., 2013). TDCS can enhance the cognitive-motor function and is beneficial for multiple neurological and psychiatric disorders (e.g., depression and Alzheimer's disease) (Kuo et al., 2014; Summers et al., 2016).

In recent years, researchers have explored the effects of tDCS on the physical performance of healthy individuals. Anodal tDCS applied over the primary motor cortex (M1) can improve multiple physical performances, such as balance control (Saruco, 2017; de Moura et al., 2019), pain perception (Vaseghi et al., 2014), muscle strength, and muscular endurance (Lattari et al., 2018; Vargas et al., 2018; Machado, S. et al., 2019). Specifically, tDCS designed to target the sensorimotor region has induced improvements in the physical performances of the foot and ankle of healthy adults (Devanathan and Madhavan, 2016; Zhou et al., 2018). For example, Zhou et al. (2018) observed that one session of tDCS targeting M1 has improved the vibrotactile sensation of the foot sole of healthy older adults when standing. These efforts have shed a light on a novel strategy for the enhancement of the physical performances of the foot and ankle by using tDCS to modulate the excitability of cortical brain regions. Considering the different methodologies and unpredicted effects, systematic review of published studies can provide valuable summaries of the effects of tDCS on the physical performances of the foot and ankle.

This study thus aims to systematically review available peerreviewed publications to date on the effects of tDCS on the physical performances of the foot and ankle of healthy adults. We then further discuss the underlying neurophysiological mechanism through which cortical activities influence the neuromechanical management of physical performances of the foot and ankle. This review will provide the most recent achievements and a better understanding of research efforts in this direction to ultimately help optimize the implementation of tDCS to enhance the physical performances of the foot and ankle in the near future.

\section{METHODS}

The method of this review was designed following the recommendations of the Preferred Reporting Items for Systematic reviews and Meta-Analyses and the Cochrane Handbook for Systematic Reviews of Interventions (Moher et al., 2009; Cumpston et al., 2019).

\section{Search Strategy}

This systematic review conducted a comprehensive search of three databases, namely, PubMed, Web of Science, and EBSCO, up to May 2020. The search was performed using the terms "foot," "toe," and "ankle," which were separately combined with "transcranial direct current stimulation" or "tDCS" in all databases. Boolean operators "AND" and "OR" were used to combine keywords according to the recommendations of each database. All results found in the search were imported into the EndNote reference manager (EndNote X9, USA, Stanford) to gather together and automatically find out duplicate records.

\section{Eligibility Criteria and Article Selection}

The following inclusion criteria were applied based on the Participants, Intervention, Comparison, Outcomes, and Study design (Ferreira et al., 2019). (1) The participants were healthy adults with no history of musculoskeletal injury and overt neurological disease. (2) The intervention was tDCS, regardless of stimulation types, stimulus intensity, duration, and electrode location. (3) A comparison was with sham tDCS (i.e., placebo). (4) The primary outcomes were strength, perception, flexibility, or other related variables of the foot and ankle. (5) The study design was randomized, crossover, and sham-control designs. Animal studies and non-English studies were excluded. Reviews, case reports, letters, opinions, and conference abstracts were also excluded (Figure 1).

Two researchers independently evaluated the results of the search and resolved differences through discussion (SL and $\mathrm{BF})$. The Abstracts and full texts of relevant articles were read thoroughly, and only those that met the eligibility criteria were selected. Then, the researchers further confirmed the selected articles and discussed possible disagreements; if any disagreement persisted, then a third researcher was consulted and judged the results (WJ).

\section{Data Extraction}

The original data of the included articles were summarized in Microsoft Excel (Microsoft Corporation, Redmond, WA, USA). The findings were divided into two categories, namely, the effects of tDCS on the physical performances of (1) the foot and (2) ankle, to facilitate the interpretation of the results. Besides, the following data were summarized: author, sample size, gender, age, anodal/cathodal location, electrode size, current intensity, duration, control, and main outcomes. 


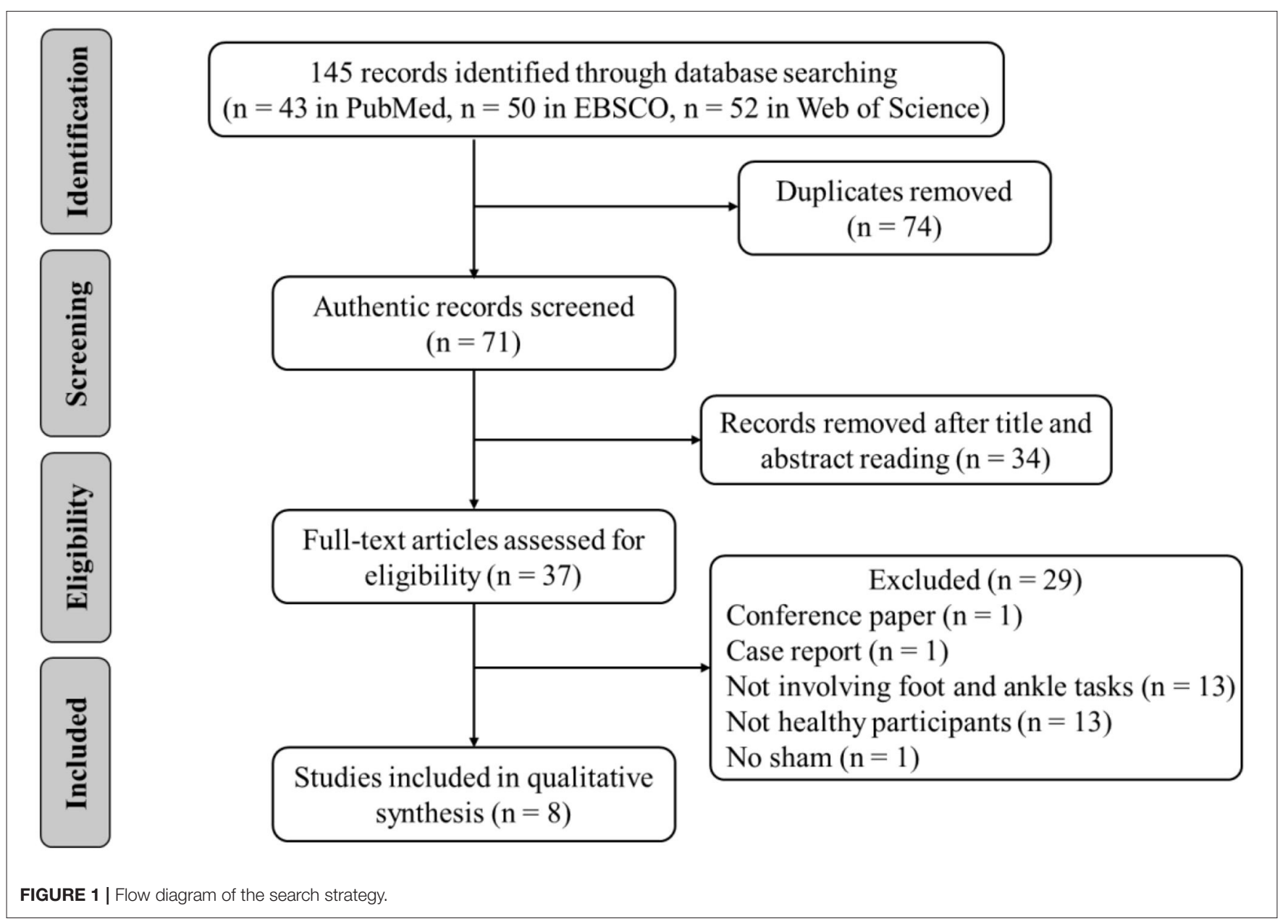

\section{Quality and Risk-of-Bias Assessments}

The Physiotherapy Evidence Database (PEDro) scale was used to assess the quality of each study (Maher et al., 2003). Studies with a PEDro score of $<6$ were deemed as having low quality.

The risk of bias of each study was assessed using the Collaboration's "Risk of Bias" tool, version 5.2 based on the Cochrane Handbook for Systematic Reviews of Interventions (Cumpston et al., 2019). The risk of bias for each study was judged as "low," "high," or "unclear" risk of bias. The two researchers independently evaluated the PEDro score and risk of bias of each study; a third researcher was consulted to reach a final consensus if any disagreement persisted.

\section{RESULTS}

A total of 145 related articles were found in the databases (43 in PubMed, 50 in EBSCO, and 52 in Web of Science). Only eight articles were included for systematic review after removing duplicate articles and excluding irrelevant studies by reading the titles, Abstracts, and full texts. Four studies examined the effects of tDCS on foot physical performance (Table 1), and the other four studied its effects on ankle physical performance (Table 3 ).

\section{Effects of tDCS on Foot Physical Performance}

Four studies investigated the effect of tDCS on foot physical performance. Fifty-four participants, consisting of 52 males and 2 females, were recruited with an age of between 20 and 61 years (Table 1). A bias in gender was observed across these studies.

Only the immediate effects of one-session tDCS were examined in these studies. Three studies used conventional tDCS applied over the sensorimotor cortex with an electrode size of $35 \mathrm{~cm}^{2}$ (Tanaka et al., 2009; Zhou et al., 2018; Yamamoto et al., 2020). One study used a $4 \times 1$ ring high-definition tDCS (HDtDCS), in which the anodal electrode was placed over $\mathrm{Cz}$ and was surrounded by four cathodal electrodes with a size of $1 \mathrm{~cm}^{2}$ (Xiao et al., 2020). Three studies applied the current intensity of $2 \mathrm{~mA}$ (Tanaka et al., 2009; Zhou et al., 2018; Xiao et al., 2020), and one study applied the intensity of $1.5 \mathrm{~mA}$ (Yamamoto et al., 2020). The duration of tDCS was $10 \mathrm{~min}$ in two studies (Tanaka et al., 2009; Yamamoto et al., 2020) and $20 \mathrm{~min}$ in the other two studies (Zhou et al., 2018; Xiao et al., 2020). The current density at the stimulation electrode was 0.043 or $0.057 \mathrm{~mA} / \mathrm{cm}^{2}$ with a total charge between 0.026 and $0.069 \mathrm{C} / \mathrm{cm}^{2}$. Sham was used as a control in these studies, in which the placements of electrodes 
TABLE 1 | The characteristics of studies investigating the effect of tDCS on foot physical performance.

\begin{tabular}{|c|c|c|c|c|c|c|c|c|}
\hline Study & Sample & Gender & Age (years) & $\begin{array}{l}\text { Drop-outs } \\
\text { (N) }\end{array}$ & Design & Task & $\begin{array}{l}\text { Cortical } \\
\text { excitability } \\
\text { examined }\end{array}$ & $\begin{array}{l}\text { PEDro } \\
\text { score }\end{array}$ \\
\hline Xiao et al. (2020) & 14 & 14 males & $22.8 \pm 1.2$ & None & Crossover & $\begin{array}{c}\text { Foot flexor strength } \\
\text { task }\end{array}$ & No & 9 \\
\hline Tanaka et al. (2009) & 10 & $\begin{array}{l}8 \text { males } \\
2 \text { females }\end{array}$ & $20-35$ & 3 & Crossover & Toe pinch force task & No & 6 \\
\hline
\end{tabular}

TABLE 2 | Stimulation protocols and main outcomes of studies investigating the effect of tDCS on foot physical performance.

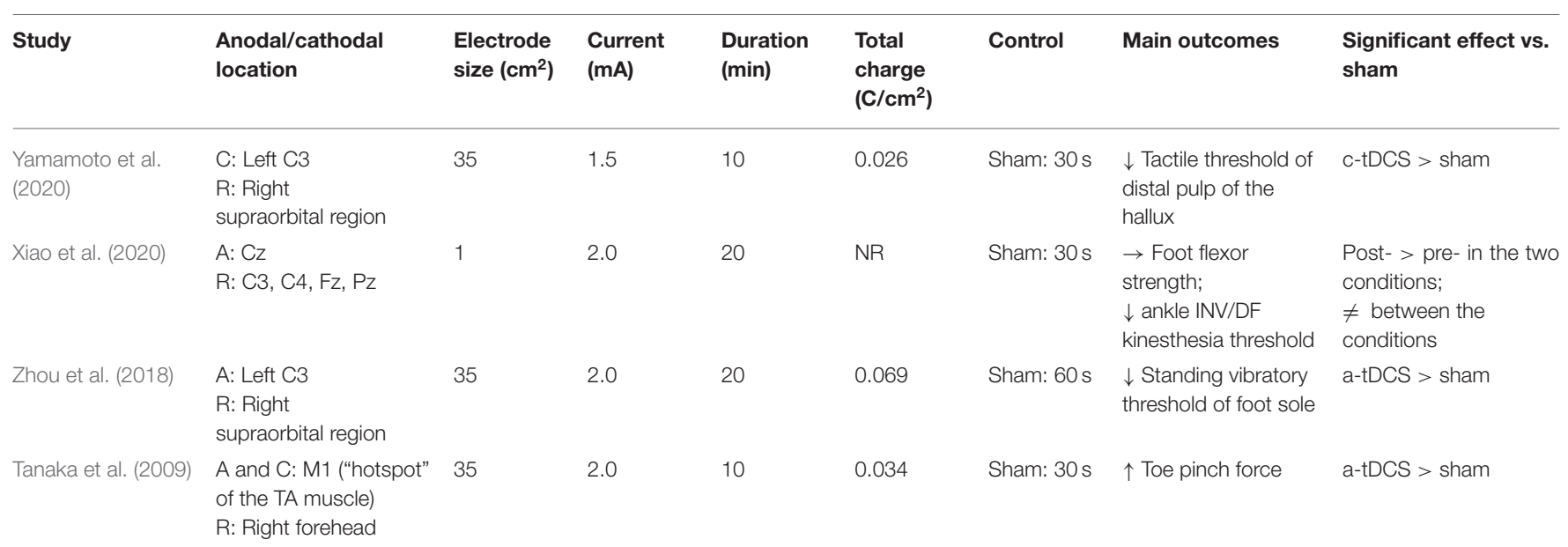

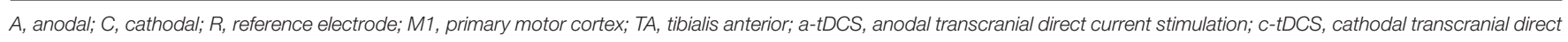

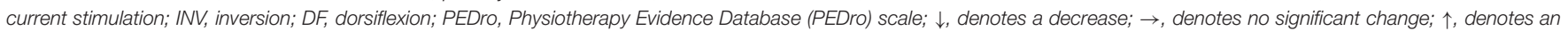
increase; C3, C4, Cz, Fz, Pz: the electrodes placement of the 10/20 EEG system; NR, not reported.

TABLE 3 | The characteristics of studies investigating the effect of tDCS on ankle physical performance.

\begin{tabular}{|c|c|c|c|c|c|c|c|c|}
\hline Study & Sample & Gender & Age (years) & $\begin{array}{l}\text { Drop-outs } \\
\text { (N) }\end{array}$ & Design & Task & $\begin{array}{c}\text { Cortical } \\
\text { excitability } \\
\text { examined }\end{array}$ & $\begin{array}{l}\text { PEDro } \\
\text { score }\end{array}$ \\
\hline $\begin{array}{l}\text { Mizuno and Aramaki, } \\
(2017)\end{array}$ & 10 & 10 males & $25 \pm 3$ & 2 & Crossover & $\begin{array}{c}\text { Passive dorsiflexion } \\
\text { task }\end{array}$ & No & 9 \\
\hline $\begin{array}{l}\text { Devanathan and } \\
\text { Madhavan, (2016) }\end{array}$ & 14 & $\begin{array}{l}6 \text { males } \\
8 \text { females }\end{array}$ & 20-32 & None & Crossover & $\begin{array}{c}\text { Ankle reaction time } \\
\text { task }\end{array}$ & No & 9 \\
\hline Sriraman et al. (2014) & 12 & $\begin{array}{l}4 \text { males, } \\
8 \text { females }\end{array}$ & 20-32 & None & Crossover & Ankle motor task & Yes & 6 \\
\hline Shah et al. (2013) & 8 & $\begin{array}{l}5 \text { males, } \\
3 \text { females }\end{array}$ & $18-26$ & None & Crossover & $\begin{array}{c}\text { Ankle visuomotor } \\
\text { task }\end{array}$ & Yes & 6 \\
\hline
\end{tabular}

were the same as real tDCS but the current was delivered in only the first 30 or $60 \mathrm{~s}$ of the stimulation (Table 2).

Several variables were assessed in these studies, including foot sole standing vibratory threshold, foot tactile threshold, foot flexor strength, and toe pinch force. Specifically, Zhou et al. (2018) observed that the standing vibratory threshold was decreased (i.e., better sensation) after anodal tDCS compared with sham. Yamamoto et al. (2020) examined the effects of cathodal tDCS designed to target the left motor area on foot tactile threshold and observed a significant decrease in the tactile threshold of the left center of the distal pulp of the hallux after cathodal tDCS compared with sham. One study showed that tDCS could improve toe pinch force (Tanaka et al., 2009). More recently, Xiao et al. (2020) found no significant differences in foot flexor strength between anodal HD-tDCS and sham stimulation. 


\section{Effects of tDCS on Ankle Physical Performance}

Four studies examined the effects of tDCS on ankle physical performance. Forty-four participants, consisting of 25 males and 19 females, were recruited. The age of the participants was between 18 and 32 years (Table 3 ).

Only the immediate effects of one-session tDCS were examined in these studies. The target of tDCS was M1 in three studies (Sriraman et al., 2014; Devanathan and Madhavan, 2016; Mizuno and Aramaki, 2017), and the targets in the other study were M1 and the cerebellum (Shah et al., 2013). Three studies used transcranial magnetic stimulation to identify the area of M1 by hot spotting for the left tibialis anterior (TA) muscle (Shah et al., 2013; Sriraman et al., 2014; Devanathan and Madhavan, 2016), and one study placed the electrodes according to the 10/20 EEG system (Mizuno and Aramaki, 2017). The electrode sizes were between 8 and $35 \mathrm{~cm}^{2}$ (Shah et al., 2013; Sriraman et al., 2014; Devanathan and Madhavan, 2016; Mizuno and Aramaki, 2017). Three studies applied the intensity of $1 \mathrm{~mA}$ for $15 \mathrm{~min}$ (Shah et al., 2013; Sriraman et al., 2014; Devanathan and Madhavan, 2016) and the other one used $2 \mathrm{~mA}$ for $10 \mathrm{~min}$ (Mizuno and Aramaki, 2017). The current density at the stimulation electrode was $0.057,0.08$, or $0.125 \mathrm{~mA} / \mathrm{cm}^{2}$ with a total charge between 0.034 and $0.113 \mathrm{C} / \mathrm{cm}^{2}$. Sham was used as a control in these studies, in which the placement of electrodes was the same as real tDCS but the current was delivered in only the first $30 \mathrm{~s}$ of the stimulation.

For the excitability assessment induced by tDCS, one study showed that cerebellar cathodal and anodal tDCS increased the normalized motor-evoked potential (MEP) amplitudes for the TA muscle compared to the sham condition (Shah et al., 2013). The other study observed a trend toward a greater change in MEP amplitude during anodal tDCS compared to the pre-stimulation (Sriraman et al., 2014) (Table 4).

Ankle physical performance outcomes, including ankle reaction time, ankle range of motion, accuracy index for the ankle motor task, and passive kinesthesia thresholds, were measured in these studies. Devanathan and Madhavan (2016) observed that anodal tDCS could reduce the choice reaction time of ankle dorsiflexion compared with a sham condition. Mizuno and Aramaki (2017) observed that cathodal tDCS significantly increased ankle range of motion. Shah et al. (2013) and Sriraman et al. (2014) explored the effects of tDCS on ankle motor skill learning. The former found that anodal cerebellar, cathodal cerebellar, and anodal M1 tDCSs improved the target-tracking accuracy index of the ankle; and the latter similarly observed that $\mathrm{tDCS}$ increased motor performance during the practice of a skilled ankle motor task. Xiao et al. (2020) observed that anodal HD-tDCS decreased the passive kinesthesia thresholds of ankle inversion and dorsiflexion from pre- to post-stimulation, but no significant differences were observed between anodal and sham HD-tDCS.

\section{Risk of Bias and Quality of Evidence}

The summary risk of bias graph was illustrated in Figure 2. One study maintained a low risk of bias in all the domains evaluated
(Xiao et al., 2020), whereas the other studies showed a certain high or uncertain risk in the risk-of-bias assessment. Regarding random sequence generation, all studies utilized randomization, but only one study sufficiently described the methods used for random sequence generation (Xiao et al., 2020). For blinding, five studies used a double-blind approach, one utilized single blinding, and the remaining described that no one noticed the difference between groups. Only two studies presented a high risk of incomplete outcome data. Three participants did not perform the hand pinch force task because of machine trouble and some other reasons in Tanaka et al. (2009). Two participants were excluded based on the results of the SmirnovGrubbs rejection test by (Mizuno and Aramaki, 2017). Regarding selective reporting, all studies were deemed as having a low risk of bias. Besides, the PEDro scores of all the studies were over six, which indicated that all the included studies have high quality. Generally, the studies performed well in terms of allocation, blinding effectiveness, selective reporting, avoidance of order effects, maintenance of stimulation well-tolerated, and absence of side effects.

\section{DISCUSSION}

In this study, we systematically reviewed the literature reporting the effects of tDCS on the physical performances of the foot and ankle. Seven of the eight included studies showed improvements in the task performances of the foot and ankle, such as motor performance, motor learning skill, and somatosensory function, which suggests that tDCS is promising to help improve the physical performances of the foot and ankle. However, due to the small sample size, various tDCS parameters, etc., these findings still need to be examined and confirmed in future studies. We will thus discuss the underlying mechanism and outlook on the possible directions of futures studies.

Nowadays, the effectiveness of tDCS is gradually certified in improving physical performances (Vargas et al., 2018; Lattari et al., 2020a,b). The effects of tDCS on lower limb performance, especially the physical performances of the foot and ankle, are relatively understudied to date (Fleming et al., 2017). Applying tDCS over the foot and ankle area of the motor cortex is challenging because of its deep position in the interhemispheric fissure (Foerster et al., 2018b). However, an initial study exploring the effects of tDCS on lower limb M1 excitability reported that $10 \mathrm{~min}$ of $2 \mathrm{~mA}$ anodal conventional tDCS with an electrode size of $35 \mathrm{~cm}^{2}$ can increase the corticospinal excitability of the leg area of the motor cortex as reflected by an increase in the amplitude of motor-evoked potentials of the TA muscle (Jeffery et al., 2007). In line with the aforementioned preliminary finding, subsequent studies have shown that tDCS can also modulate the excitability of the lower-limb area of the motor cortex and further alter the physical performances of the foot and ankle of healthy adults and thus has potential benefits on promoting the physical performances of the foot and ankle (Tanaka et al., 2009; Shah et al., 2013; Sriraman et al., 2014).

Notably, high variability in the effects of tDCS on these types of physical performances was observed (Angius et al., 2018). For 
TABLE 4 | Stimulation protocols and main outcomes of studies investigating the effect of tDCS on ankle physical performance.

\begin{tabular}{|c|c|c|c|c|c|c|c|c|}
\hline Study & $\begin{array}{l}\text { Anodal/cathodal } \\
\text { location }\end{array}$ & $\begin{array}{l}\text { Electrode } \\
\text { size }\left(\mathrm{cm}^{2}\right)\end{array}$ & $\begin{array}{l}\text { Current } \\
(\mathrm{mA})\end{array}$ & $\begin{array}{l}\text { Duration } \\
\text { (min) }\end{array}$ & $\begin{array}{l}\text { Total } \\
\text { charge } \\
\left(\mathrm{C} / \mathrm{cm}^{2}\right)\end{array}$ & Control & Main outcomes & $\begin{array}{l}\text { Significant effect vs. } \\
\text { sham }\end{array}$ \\
\hline $\begin{array}{l}\text { Mizuno and } \\
\text { Aramaki, (2017) }\end{array}$ & $\begin{array}{l}\text { A and C: } \mathrm{Cz} \\
\mathrm{R}: \text { Center of the forehead }\end{array}$ & 35 & 2.0 & 10 & 0.034 & Sham: $30 \mathrm{~s}$ & $\begin{array}{l}\uparrow \text { Ankle range of } \\
\text { motion }\end{array}$ & c-tDCS > sham \\
\hline $\begin{array}{l}\text { Devanathan and } \\
\text { Madhavan, (2016) }\end{array}$ & $\begin{array}{l}\text { A: M1 ("hotspot" of the } \\
\text { TA muscle) } \\
\text { R: Right } \\
\text { supraorbital region }\end{array}$ & 12.5 & 1.0 & 15 & 0.072 & Sham: $30 \mathrm{~s}$ & $\begin{array}{l}\downarrow \text { Ankle dorsiflexion } \\
\text { choice reaction time }\end{array}$ & a-tDCS > sham \\
\hline $\begin{array}{l}\text { Sriraman et al. } \\
(2014)\end{array}$ & $\begin{array}{l}\text { A: M1 ("hotspot" of the } \\
\text { TA muscle) } \\
\text { R: Right } \\
\text { supraorbital region }\end{array}$ & 8 & 1.0 & 15 & 0.113 & Sham: $30 \mathrm{~s}$ & $\begin{array}{l}\uparrow \text { Accuracy index of } \\
\text { ankle tracking }\end{array}$ & a-tDCS > sham \\
\hline Shah et al. (2013) & $\begin{array}{l}\text { A and C: M1 ('hotspot' of } \\
\text { the TA muscle), left } \\
\text { cerebellum } \\
\text { R: Right supraorbital } \\
\text { region, left } \\
\text { buccinator muscle }\end{array}$ & 8 & 1.0 & 15 & 0.113 & Sham: $30 \mathrm{~s}$ & $\begin{array}{l}\uparrow \text { Accuracy index of } \\
\text { ankle tracking }\end{array}$ & $\begin{array}{l}\text { a-tDCS > sham } \\
\text { cerebellar a-tDCS > } \\
\text { sham } \\
\text { cerebellar c-tDCS } \\
>\text { sham }\end{array}$ \\
\hline
\end{tabular}

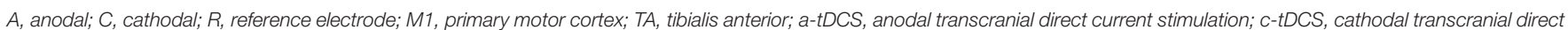
current stimulation; PEDro, Physiotherapy Evidence Database (PEDro) scale; $\downarrow$, denotes a decrease; $\uparrow$, denotes an increase; Cz, the electrodes placement of the 10/20 EEG system.

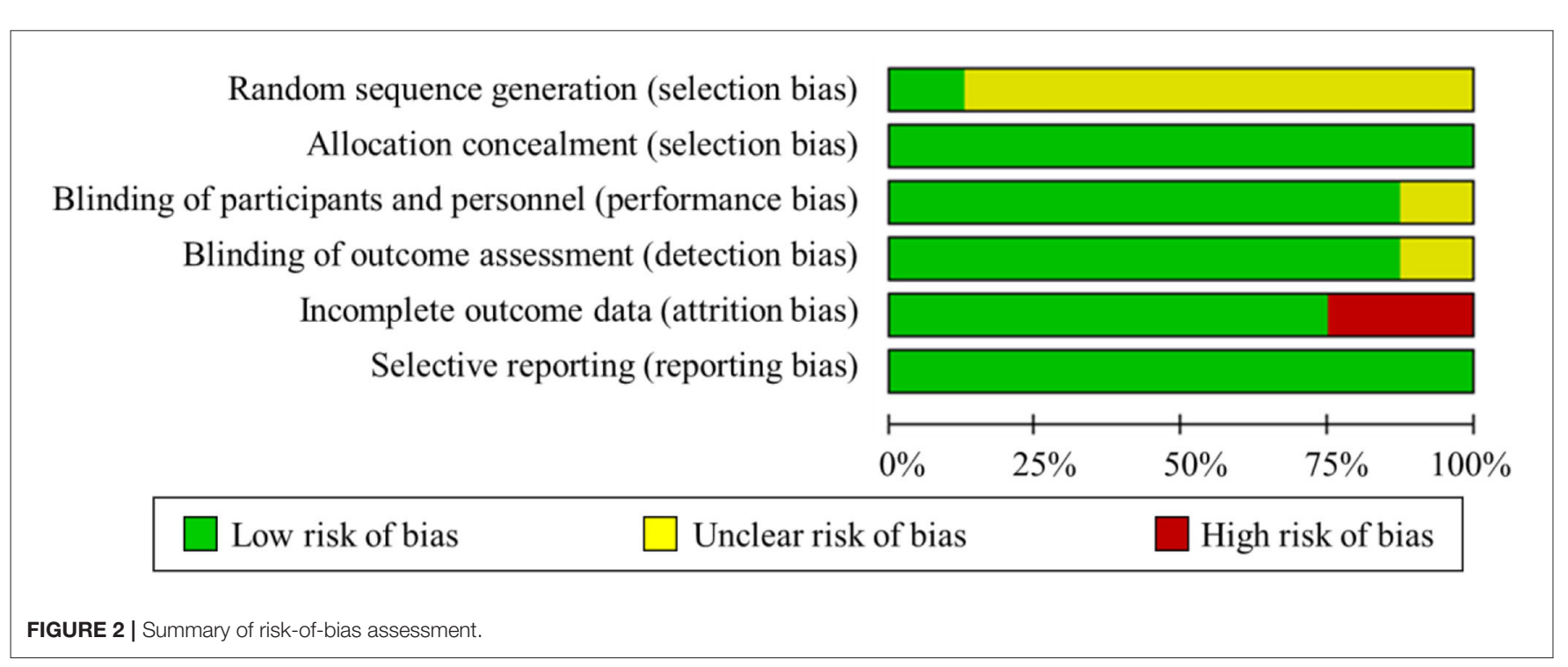

example, anodal tDCS failed to enhance the muscle strength of the lower extremity of healthy participants, including knee extensor and foot flexor strength (Flood et al., 2017; Maeda et al., 2017; Xiao et al., 2020). The variability may have arisen from the variance in the characteristics of the participants, the design of study protocols, and tDCS montages. Specifically, the variance in experimental outcomes in response to tDCS was caused by several factors associated with high inter-individual variability, including the organization of local circuits, basal level of function, psychological state, level of neurotransmitters and receptor sensibility, baseline neurophysiological state, and genetic aspects (López-Alonso et al., 2015; Pellegrini et al., 2018a,b; Machado et al., 2019). In addition, those previous studies used different experimental designs and stimulation protocols (i.e., different montages), potentially contributing to the variance in the results or even the reversal effect (Datta et al., 2009; Angius et al., 2018; Machado, S. et al., 2019; Hassanzahraee et al., 2020; Pellegrini et al., 2020). For example, Hassanzahraee et al. (2020) observed the reversal of corticospinal excitability of anodal tDCS with a current intensity of $1.0 \mathrm{~mA}$ when the stimulation duration was over $26 \mathrm{~min}$, which may provide certain implications for preventing excessive brain activation. Additionally, a "ceiling effect" may limit the benefit induced by tDCS in healthy adults (Zhou et al., 2018), that is, healthy participants can perform the task well at baseline; thus, the tDCS-induced improvement would be not as high as expected. Nevertheless, the main outcomes of these studies showed the potential of tDCS in enhancing the physical performances of the foot and ankle, yet its effectiveness 
should be further confirmed in future studies with more rigorous study design and larger sample sizes.

These studies showed the potential of tDCS in enhancing the physical performances of the foot and ankle. However, the montages of tDCS (e.g., current intensity, duration, and electrode size) were inconsistent across the included studies. Specifically, three studies that investigated the effect of tDCS on foot physical performance used the conventional tDCS, which delivered a current of $1.5-2 \mathrm{~mA}$ via two large sponges (size: $35 \mathrm{~cm}^{2}$ ). The total charge of received current on targets was between 0.026 and $0.069 \mathrm{C} / \mathrm{cm}^{2}$, which can adequately induce variation in the response threshold of the stimulated neurons and change the electrical activity of the neurons (Fertonani and Miniussi, 2017). Thus, Zhou et al. (2018) found that $20 \mathrm{~min}$ of $2 \mathrm{~mA}$ tDCS applying over $\mathrm{C} 3 \mathrm{can}$ also improve foot somatosensory function. Similarly, among the studies investigating the effect of tDCS on ankle physical performance, four studies used the conventional tDCS delivering the current intensity between 1 and $2 \mathrm{~mA}$ via two large electrodes with different sizes (i.e., $8-35 \mathrm{~cm}^{2}$ ). The total current charge was between 0.034 and $0.113 \mathrm{C} / \mathrm{cm}^{2}$, and these studies showed that applying tDCS over M1 could increase ankle motor performance. However, this conventional tDCS may induce diffuse current flow on targets and the distribution of the induced electrical field may vary across participants. More importantly, the return of current in this conventional tDCS cannot be well-controlled, and may thus induce the inhibitory effects on the cortical regions next to the targeted area (To et al., 2016). For example, Zhou et al. (2018) targeted the primary sensory cortex (C3 area of 10/20 EEG template) using large sponge electrodes, and the excitability of the primary motor cortex (located anatomically next to the primary sensory cortex) may be inhibited, which may thus limit the effects of tDCS on the functional performance (e.g., they did not observe the significant improvement in the performance of timed-up-andgo test of mobility, though the foot-sole tactile sensation was improved). Besides, only one study using HD-tDCS observed a significant improvement in foot physical performance from pre- to post-stimulation, but not between anodal and sham HD-tDCS (Xiao et al., 2020). For anodal HD-tDCS, the anodal electrode was set at $2 \mathrm{~mA}$, while the four return electrodes placed $3.5 \mathrm{~cm}$ apart from the anodal electrode were programmed to $-0.5 \mathrm{~mA}$. The previous study anticipated that $-0.25 \mathrm{~mA}$ inhibitory input from the surrounding cortical areas may negate or override the focalized $1 \mathrm{~mA}$ current intensity over M1, which may influence overall M1 excitability (Pellegrini et al., 2020). Thus, the improvements in foot physical performance induced by anodal HD-tDCS may not be significant (Xiao et al., 2020). The applicable HD-tDCS protocol and its effectiveness should be further confirmed in future studies with more rigorous study design and larger sample size.

The mechanisms of tDCS in improving the physical performances of the foot and ankle are still uncertain (Angius et al., 2017). However, several possible explanations have been made. The modulation of the cortical excitability and the activation of the synaptic neuroplasticity of targeting brain regions play a key role in the regulation of neural circuits responsible for biomechanical management (Nitsche et al.,
2008). Specifically, the current flow delivered by tDCS may augment active synaptic connections between the neuronal structures of cortical regions and thus may induce sustained changes in the neural activity of motor neurons. Such neuronal changes can improve the degree of the synchronous discharge of motor units, which influence the neural circuitry on the neuromechanical regulation of the foot and ankle and then improve their physical performance (Bindman et al., 1964; Dutta et al., 2015; Patel et al., 2019). TDCS can also induce an increase in cortico-muscular coherence, which is defined as the coherence mapping the synchrony between cortical neural activity and muscles (Chen C. et al., 2019). This occurrence may also support the aforementioned hypothesis of the underlying mechanism. Besides, the neuronal circuits involved in the regulation of motor tasks are likely active at a heightened state (Ziemann et al., 2004; Sriraman et al., 2014; Chen J. et al., 2019). The tDCS-induced improvements in physical performance and/or motor learning skills are commonly observed in the presence of activity-dependent modifications of synapses. Thus, the membrane shifting induced by anodal tDCS accessibly may shape synaptic plasticity and improve motor performance and learning (Sriraman et al., 2014). Sensory sensitivity depends on the degree of excitation in the somatosensory cortex and the integrity of the individual's neural circuitry (Fregni and Pascual-Leone, 2007). Wang et al. (2015) observed that anodal tDCS could modulate and augment cortical responsiveness to foot pressure stimuli in healthy adults by increasing the activation of the left posterior paracentral lobule (including the primary sensory cortex [S1]), and this tDCS-induced increase in cortical responsiveness to pressure stimuli may help the sensation of the lower limbs. Additionally, $\mathrm{S} 1$ is next to M1; therefore, tDCS targeting S1 may also increase the excitability of M1. Thus, improvements in sensory function may be attributed to the tDCS-induced activation of M1.

Interestingly, cathodal tDCS applied over the motor area also induced a positive effect on foot tactile sensation and ankle range of motion (Mizuno and Aramaki, 2017; Yamamoto et al., 2020). Cathodal tDCS usually decreases cortical excitability. By contrast, the administration of cathodal tDCS to the motor cortex of one hemisphere increases the excitability of the motor cortex of the other hemisphere in healthy participants (Schambra et al., 2003) and improves the physical performance of the ipsilateral foot (Yamamoto et al., 2020). Besides, an increase in ankle range of motion may be based on decreased pain perception secondary to the decreased excitability of the cerebral cortex caused by cathodal tDCS (Mizuno and Aramaki, 2017). Although anodal and cathodal tDCS maintain discrepant neurophysiological effects, both have shown benefits in improving the physical performance of the foot and ankle.

Multiple intermediate structural and functional levels between cortical regions and the foot and ankle in the regulatory pathway of the physical functions of the foot and ankle may mediate and integrate the effects of tDCS (Fertonani and Miniussi, 2017). These components are interconnected over multiple levels; therefore, tDCS that modulates neurons 
close to the discharge threshold, which are potentially engaged in the execution of a specific task, may also regulate the functionality of multiple components (e.g., task-related networks and secondary-level neural circuitry) in the regulatory system (Siebner et al., 2009; Bikson et al., 2013; Miniussi et al., 2013; Luft et al., 2014). However, some improvements in performance have been noted without remarkable changes in corticospinal excitability (Abdelmoula et al., 2016; Angius et al., 2016). Thus, characterizing the multi-level components of the regulatory system of the foot and ankle's physical function in future studies using neuroimaging techniques will ultimately advance the understanding of the underlying mechanisms behind the ergogenic neuromechanical effects of tDCS on the foot and ankle, as well as the other parts of the body extremities.

The results of this systematic review should also be taken with caution since the included studies have some methodological limitations. First, the sample size of the included studies in this review is relatively small. Second, the selection of tDCS parameters varied across studies, and many of them lacked optimization. For example, two studies applied the conventional tDCS over the C3 area, which may not be the appropriate target for the control of the lower-extremity function (Zhou et al., 2018; Yamamoto et al., 2020). Thus, a standardized protocol of tDCS using a more rigorous study design and advanced neuro-modeling technique is highly demanded in future studies aiming at examining the effectiveness of tDCS on the physical performances of the foot and ankle. Besides, the neurophysiological mechanisms through which tDCS benefits the physical performance are still not fully understood. Only two studies measured the changes of cortical excitability within the tDCS targets (i.e., M1), but the "dose-response" effects of tDCS were not examined (Shah et al., 2013; Sriraman et al., 2014). Further studies are warranted to estimate the on-target "does" of tDCS (e.g., the normal component of the electric field within the tDCS targets) using neuro-modeling techniques with the brain magnetic resonance images of participants and correlate such dose to the observed functional improvements (Fischer et al., 2017). This will provide fundamental knowledge into the causal relationship between the tDCS-induced neurophysiological changes of cortical regions and the changes in behavior induced by tDCS. A bias in gender was observed in the studies investigated the effects of tDCS on foot sensory function and foot muscle strength. However, to date, no studies have examined how gender influences the tDCS-induced effects on foot physical performance, which is worthwhile to be explored in the future. Besides, much more numbers of studies generally demonstrated that tDCS can induce benefits to functional performance. However, potential publication bias should be noted, that is, studies with positive results are more preferred to be reported. Negative results on the efficacy of tDCS might be encouraged to be published to critique the implementation of tDCS, which will ultimately help optimize the protocol of tDCS intervention.
Research efforts on exploring the effects of tDCS on the physical performances of the foot and ankle have emerged recently, and a relatively limited number of studies $(n=8)$ was published. The current review was thus performed based upon this number of publications. Besides, this study only focused on the effect of tDCS on the physical performances of the foot and ankle of healthy adults; patients with diminished or impaired foot and ankle functionality should be taken into account in future studies.

\section{CONCLUSION}

Based on the existing studies, tDCS is promising to help improve the physical performances of the foot and ankle, such as motor performance, motor learning skill, and somatosensory function, which needs to be examined and confirmed in future studies. The underlying mechanism has been postulated that tDCS increased neuronal excitability of the targeted cortex and may potentially enhance the neuromechanical management of the physical performances of the foot and ankle. However, future studies with a larger sample size and a more rigorous experimental design (i.e., study protocol and tDCS montage) are warranted to confirm the findings of current studies and explore the neurophysiological and neuromechanical mechanisms of tDCSinduced improvements in the physical performances of the foot and ankle.

\section{DATA AVAILABILITY STATEMENT}

The original contributions presented in the study are included in the article/supplementary materials, further inquiries can be directed to the corresponding authors.

\section{AUTHOR CONTRIBUTIONS}

SX contributed to study design, data collection, drafting, and revising the manuscript. JZ and WF contributed to supervising study design, completing data analysis and interpretation, and revising the manuscript. BW and XZ revised the manuscript. All authors have read and approved the final version of the manuscript and agree with the order of the presentation of the authors.

\section{FUNDING}

This research was funded by the National Key Technology Research and Development Program of the Ministry of Science and Technology of China (2019YFF0302100, 2018YFF0300500), the National Natural Science Foundation of China (11772201, 119320131), Talent Development Fund of Shanghai Municipal (2018107), and the Dawn Program of Shanghai Education Commission, China (19SG47). 


\section{REFERENCES}

Aagaard, P. (2018). Spinal and supraspinal control of motor function during maximal eccentric muscle contraction: effects of resistance training. J. Sport Health Sci. 7, 282-293. doi: 10.1016/j.jshs.2018.06.003

Abdelmoula, A., Baudry, S., and Duchateau, J. (2016). Anodal transcranial direct current stimulation enhances time to task failure of a submaximal contraction of elbow flexors without changing corticospinal excitability. Neuroscience 322, 94-103. doi: 10.1016/j.neuroscience.2016.02.025

Angius, L., Hopker, J., and Mauger, A. R. (2017). The ergogenic effects of transcranial direct current stimulation on exercise performance. Front. Physiol. 8:90. doi: 10.3389/fphys.2017.00090

Angius, L., Pageaux, B., Hopker, J., Marcora, S. M., and Mauger, A. R. (2016). Transcranial direct current stimulation improves isometric time to exhaustion of the knee extensors. Neuroscience 339, 363-375. doi: 10.1016/j.neuroscience.2016.10.028

Angius, L., Pascual-Leone, A., and Santarnecchi, E. (2018). Brain stimulation and physical performance. Prog. Brain Res. 240, 317-339. doi: 10.1016/bs.pbr.2018.07.010

Bikson, M., Name, A., and Rahman, A. (2013). Origins of specificity during tDCS: anatomical, activity-selective, and input-bias mechanisms. Front. Hum. Neurosci. 7:688. doi: 10.3389/fnhum.2013.00688

Bindman, L. J., Lippold, O. C., and Redfearn, J. W. (1964). The action of brief polarizing currents on the cerebral cortex of the rat (1) during current flow and (2) in the production of long-lasting after-effects. J. Physiol. 172, 369-382. doi: 10.1113/jphysiol.1964.sp007425

Chen, C., Fang, Y., Wang, X., Bao, S. C., Tang, Z., and Tong, R. K. (2019). Excitation comparison between multi-site stimulation using network-based tDCS and focal stimulation using high-definition tDCS. Conf. Proc. IEEE Eng. Med. Biol. Soc. 2019, 6884-6887. doi: 10.1109/EMBC.2019.8857287

Chen, J., Li, Y., Zhang, G., Jin, X., Lu, Y., and Zhou, C. (2019). Enhanced inhibitory control during re-engagement processing in badminton athletes: an event-related potential study. J. Sport Health Sci. 8, 585-594. doi: 10.1016/j.jshs.2019.05.005

Cumpston, M., Li, T., Page, M. J., Chandler, J., Welch, V. A., Higgins, J. P., et al. (2019). Updated guidance for trusted systematic reviews: a new edition of the Cochrane Handbook for Systematic Reviews of Interventions. Cochrane Database Syst. Rev. 10:Ed000142. doi: 10.1002/14651858.ED000142

Datta, A., Bansal, V., Diaz, J., Patel, J., Reato, D., and Bikson, M. (2009). Gyriprecise head model of transcranial direct current stimulation: improved spatial focality using a ring electrode versus conventional rectangular pad. Brain Stimul. 2, 201-207. doi: 10.1016/j.brs.2009.03.005

de Moura, M. C. D. S., Hazime, F. A., Marotti Aparicio, L. V., Grecco, L. A. C., Brunoni, A. R., and Hasue, R. H. (2019). Effects of transcranial direct current stimulation (tDCS) on balance improvement: a systematic review and meta-analysis. Somatosens. Mot. Res. 36, 122-135. doi: 10.1080/08990220.2019.1624517

Devanathan, D., and Madhavan, S. (2016). Effects of anodal tDCS of the lower limb M1 on ankle reaction time in young adults. Exp. Brain Res. 234, 377-385. doi: $10.1007 / \mathrm{s} 00221-015-4470-y$

Dutta, A., Krishnan, C., Kantak, S. S., Ranganathan, R., and Nitsche, M. A. (2015). Recurrence quantification analysis of surface electromyogram supports alterations in motor unit recruitment strategies by anodal transcranial direct current stimulation. Restor. Neurol. Neurosci. 33, 663-669. doi: $10.3233 / \mathrm{RNN}-140469$

Ferreira, N. R., Junqueira, Y. N., Corrêa, N. B., Fonseca, E. O., Brito, N. B. M., Menezes, T. A., et al. (2019). The efficacy of transcranial direct current stimulation and transcranial magnetic stimulation for chronic orofacial pain: a systematic review. PLoS ONE 14:e0221110. doi: 10.1371/journal.pone.0221110

Fertonani, A., and Miniussi, C. (2017). Transcranial electrical stimulation: what we know and do not know about mechanisms. Neuroscientist 23, 109-123. doi: $10.1177 / 1073858416631966$

Fischer, D. B., Fried, P. J., Ruffini, G., Ripolles, O., Salvador, R., Banus, J., et al. (2017). Multifocal tDCS targeting the resting state motor network increases cortical excitability beyond traditional tDCS targeting unilateral motor cortex. Neuroimage 157, 34-44. doi: 10.1016/j.neuroimage.2017.05.060

Fleming, M. K., Pavlou, M., Newham, D. J., Sztriha, L., and Teo, J. T. (2017). Non-invasive brain stimulation for the lower limb after stroke: what do we know so far and what should we be doing next? Disabil. Rehabil. 39, 714-720. doi: 10.3109/09638288.2016.1161835

Flood, A., Waddington, G., Keegan, R. J., Thompson, K. G., and Cathcart, S. (2017). The effects of elevated pain inhibition on endurance exercise performance. PeerJ 5:e3028. doi: 10.7717/peerj.3028

Foerster, Á., Dutta, A., Kuo, M. F., Paulus, W., and Nitsche, M. A. (2018a). Effects of anodal transcranial direct current stimulation over lower limb primary motor cortex on motor learning in healthy individuals. Eur. J. Neurosci. 47, 779-789. doi: 10.1111/ejn.13866

Foerster, Á., Rezaee, Z., Paulus, W., Nitsche, M. A., and Dutta, A. (2018b). Effects of cathode location and the size of anode on anodal transcranial direct current stimulation over the leg motor area in healthy humans. Front. Neurosci. 12:443. doi: 10.3389/fnins.2018.00443

Fregni, F., and Pascual-Leone, A. (2007). Technology insight: noninvasive brain stimulation in neurology-perspectives on the therapeutic potential of rTMS and tDCS. Nat. Clin. Pract. Neurol. 3, 383-393. doi: 10.1038/ncpneuro0530

Hassanzahraee, M., Nitsche, M. A., Zoghi, M., and Jaberzadeh, S. (2020). Determination of anodal tDCS duration threshold for reversal of corticospinal excitability: an investigation for induction of counter-regulatory mechanisms. Brain Stimul. 13, 832-839. doi: 10.1016/j.brs.2020.02.027

Jeffery, D. T., Norton, J. A., Roy, F. D., and Gorassini, M. A. (2007). Effects of transcranial direct current stimulation on the excitability of the leg motor cortex. Exp. Brain Res. 182, 281-287. doi: 10.1007/s00221-007-1093-y

Kuo, M. F., Paulus, W., and Nitsche, M. A. (2014). Therapeutic effects of noninvasive brain stimulation with direct currents (tDCS) in neuropsychiatric diseases. Neuroimage 85, 948-960. doi: 10.1016/j.neuroimage.2013.05.117

Lattari, E., Campos, C., Lamego, M. K., Legey, S., Neto, G. M., Rocha, N. B., et al. (2020a). Can transcranial direct current stimulation improve muscle power in individuals with advanced weight-training experience? J. Strength Cond. Res. 34, 97-103. doi: 10.1519/JSC.0000000000001956

Lattari, E., Oliveira, B. R. R., Monteiro Júnior, R. S., Marques Neto, S. R., Oliveira, A. J., Maranhão Neto, G. A., et al. (2018). Acute effects of single dose transcranial direct current stimulation on muscle strength: a systematic review and meta-analysis. PLOS ONE 13:e0209513. doi: 10.1371/journal.pone.02 09513

Lattari, E., Rosa Filho, B. J., Fonseca Junior, S. J., Murillo-Rodriguez, E., Rocha, N., Machado, S., et al. (2020b). Effects on volume load and ratings of perceived exertion in individuals' advanced weight training after transcranial direct current stimulation. J. Strength Cond. Res. 34, 89-96. doi: 10.1519/JSC.0000000000002434

López-Alonso, V., Fernández-Del-Olmo, M., Costantini, A., Gonzalez-Henriquez, J. J., and Cheeran, B. (2015). Intra-individual variability in the response to anodal transcranial direct current stimulation. Clin. Neurophysiol. 126, 2342-2347. doi: 10.1016/j.clinph.2015.03.022

Luft, C. D., Pereda, E., Banissy, M. J., and Bhattacharya, J. (2014). Best of both worlds: promise of combining brain stimulation and brain connectome. Front. Syst. Neurosci. 8:132. doi: 10.3389/fnsys.2014.00132

Machado, D., Unal, G., Andrade, S. M., Moreira, A., Altimari, L. R., Brunoni, A. R., et al. (2019). Effect of transcranial direct current stimulation on exercise performance: a systematic review and meta-analysis. Brain Stimul. 12, 593-605. doi: 10.1016/j.brs.2018.12.227

Machado, S., Jansen, P., Almeida, V., and Veldema, J. (2019). Is tDCS an adjunct ergogenic resource for improving muscular strength and endurance performance? A systematic review. Front. Psychol. 10:1127. doi: 10.3389/fpsyg.2019.01127

Maeda, K., Yamaguchi, T., Tatemoto, T., Kondo, K., Otaka, Y., and Tanaka, S. (2017). Transcranial direct current stimulation does not affect lower extremity muscle strength training in healthy individuals: a triple-blind, sham-controlled study. Front. Neurosci. 11:179. doi: 10.3389/fnins.2017.00179

Maher, C. G., Sherrington, C., Herbert, R. D., Moseley, A. M., and Elkins, M. (2003). Reliability of the PEDro scale for rating quality of randomized controlled trials. Phys. Ther. 83, 713-721. doi: 10.1093/ptj/83.8.713

Miniussi, C., Harris, J. A., and Ruzzoli, M. (2013). Modelling non-invasive brain stimulation in cognitive neuroscience. Neurosci. Biobehav. Rev. 37, 1702-1712. doi: 10.1016/j.neubiorev.2013.06.014

Mizuno, T., and Aramaki, Y. (2017). Cathodal transcranial direct current stimulation over the $\mathrm{Cz}$ increases joint flexibility. Neurosci. Res. 114, 55-61. doi: 10.1016/j.neures.2016.08.004 
Moher, D., Liberati, A., Tetzlaff, J., and Altman, D. G. (2009). Preferred reporting items for systematic reviews and meta-analyses: the PRISMA statement. PLoS Med 6:e1000097. doi: 10.1371/journal.pmed.1000097

Needle, A. R., Lepley, A. S., and Grooms, D. R. (2017). Central nervous system adaptation after ligamentous injury: a summary of theories, evidence, and clinical interpretation. Sports Med. 47, 1271-1288. doi: 10.1007/s40279-016-0666-y

Nitsche, M. A., Cohen, L. G., Wassermann, E. M., Priori, A., Lang, N., Antal, A., et al. (2008). Transcranial direct current stimulation: state of the art 2008. Brain Stimul. 1, 206-223. doi: 10.1016/j.brs.2008.06.004

Nitsche, M. A., and Paulus, W. (2000). Excitability changes induced in the human motor cortex by weak transcranial direct current stimulation. J. Physiol. 527, 633-639. doi: 10.1111/j.1469-7793.2000.t01-1-00633.x

Nitsche, M. A., and Paulus, W. (2001). Sustained excitability elevations induced by transcranial DC motor cortex stimulation in humans. Neurology 57, 1899-1901. doi: 10.1212/WNL.57.10.1899

Noakes, T. D. (2011). Time to move beyond a brainless exercise physiology: the evidence for complex regulation of human exercise performance. Appl. Physiol. Nutr. Metab. 36, 23-35. doi: 10.1139/H10-082

Patel, R., Ashcroft, J., Patel, A., Ashrafian, H., Woods, A. J., Singh, H., et al. (2019). The impact of transcranial direct current stimulation on upper-limb motor performance in healthy adults: a systematic review and meta-analysis. Front. Neurosci. 13:1213. doi: 10.3389/fnins.2019.01213

Pellegrini, M., Zoghi, M., and Jaberzadeh, S. (2018a). Biological and anatomical factors influencing interindividual variability to noninvasive brain stimulation of the primary motor cortex: a systematic review and meta-analysis. Rev. Neurosci. 29, 199-222. doi: 10.1515/revneuro-2017-0048

Pellegrini, M., Zoghi, M., and Jaberzadeh, S. (2018b). Cluster analysis and subgrouping to investigate inter-individual variability to non-invasive brain stimulation: a systematic review. Rev. Neurosci. 29, 675-697. doi: 10.1515/revneuro-2017-0083

Pellegrini, M., Zoghi, M., and Jaberzadeh, S. (2020). The effects of transcranial direct current stimulation on corticospinal and cortico-cortical excitability and response variability: conventional versus high-definition montages. Neurosci Res. doi: 10.1016/j.neures.2020.06.002. [Epub ahead of print].

Rahman, A., Reato, D., Arlotti, M., Gasca, F., Datta, A., Parra, L. C., et al. (2013). Cellular effects of acute direct current stimulation: somatic and synaptic terminal effects. J. Physiol. 591, 2563-2578. doi: 10.1113/jphysiol.2012. 247171

Reardon, S. (2016). 'Brain doping' may improve athletes' performance: electrical stimulation seems to boost endurance in preliminary studies. Nature 531, 283-285. doi: 10.1038/nature.2016.19534

Rodgers, M. M. (1988). Dynamic biomechanics of the normal foot and ankle during walking and running. Phys. Ther. 68, 1822-1830. doi: $10.1093 / \mathrm{ptj} / 68.12 .1822$

Saruco, E., et al., (2017). Anodal tDCS over the primary motor cortex improves motor imagery benefits on postural control: A pilot study. Sci Rep. 7:480.

Schambra, H. M., Sawaki, L., and Cohen, L. G. (2003). Modulation of excitability of human motor cortex (M1) by $1 \mathrm{~Hz}$ transcranial magnetic stimulation of the contralateral M1. Clin. Neurophysiol. 114, 130-133. doi: 10.1016/S1388-2457(02)00342-5

Shah, B., Nguyen, T. T., and Madhavan, S. (2013). Polarity independent effects of cerebellar tDCS on short term ankle visuomotor learning. Brain Stimul. 6, 966-968. doi: 10.1016/j.brs.2013.04.008

Siebner, H. R., Hartwigsen, G., Kassuba, T., and Rothwell, J. C. (2009). How does transcranial magnetic stimulation modify neuronal activity in the brain? Implications for studies of cognition. Cortex 45, 1035-1042. doi: 10.1016/j.cortex.2009.02.007

Sriraman, A., Oishi, T., and Madhavan, S. (2014). Timing-dependent priming effects of tDCS on ankle motor skill learning. Brain Res. 1581, 23-29. doi: 10.1016/j.brainres.2014.07.021

Stagg, C. J., and Nitsche, M. A. (2011). Physiological basis of transcranial direct current stimulation. Neuroscientist 17, 37-53. doi: 10.1177/1073858410386614

Summers, J. J., Kang, N., and Cauraugh, J. H. (2016). Does transcranial direct current stimulation enhance cognitive and motor functions in the ageing brain? A systematic review and meta- analysis. Ageing Res. Rev. 25, 42-54. doi: 10.1016/j.arr.2015.11.004

Tanaka, S., Hanakawa, T., Honda, M., and Watanabe, K. (2009). Enhancement of pinch force in the lower leg by anodal transcranial direct current stimulation. Exp. Brain Res. 196, 459-465. doi: 10.1007/s00221-009-1863-9

To, W. T., Hart, J., De Ridder, D., and Vanneste, S. (2016). Considering the influence of stimulation parameters on the effect of conventional and highdefinition transcranial direct current stimulation. Expert Rev. Med. Devices 13, 391-404. doi: 10.1586/17434440.2016.1153968

Vargas, V. Z., Baptista, A. F., Pereira, G. O. C., Pochini, A. C., Ejnisman, B., Santos, M. B., et al. (2018). Modulation of isometric quadriceps strength in soccer players with transcranial direct current stimulation: a crossover study. J. Strength Cond. Res. 32, 1336-1341. doi: 10.1519/JSC.0000000000001985

Vaseghi, B., Zoghi, M., and Jaberzadeh, S. (2014). Does anodal transcranial direct current stimulation modulate sensory perception and pain? A meta-analysis study. Clin Neurophysiol. 125, 1847-1858.

Wang, Y., Hao, Y., Zhou, J., Fried, P. J., Wang, X., Zhang, J., et al. (2015). Direct current stimulation over the human sensorimotor cortex modulates the brain's hemodynamic response to tactile stimulation. Eur. J. Neurosci. 42, 1933-1940. doi: 10.1111/ejn.12953

Xiao, S., Wang, B., Zhang, X., Zhou, J., and Fu, W. (2020). Acute effects of high-definition transcranial direct current stimulation on foot muscle strength, passive ankle kinesthesia, and static balance: a pilot study. Brain Sci. 10:246. doi: 10.3390/brainsci10040246

Yamamoto, S., Ishii, D., Ichiba, N., Yozu, A., and Kohno, Y. (2020). Cathodal tDCS on the motor area decreases the tactile threshold of the distal pulp of the hallux. Neurosci. Lett. 719:133887. doi: 10.1016/j.neulet.2018.10.032

Zhou, J., Lo, O. Y., Lipsitz, L. A., Zhang, J., Fang, J., and Manor, B. (2018). Transcranial direct current stimulation enhances foot sole somatosensation when standing in older adults. Exp. Brain Res. 236, 795-802. doi: 10.1007/s00221-018-5178-6

Ziemann, U., Ilić, T. V., Pauli, C., Meintzschel, F., and Ruge, D. (2004). Learning modifies subsequent induction of long-term potentiation-like and long-term depression-like plasticity in human motor cortex. J. Neurosci. 24, 1666-1672. doi: 10.1523/JNEUROSCI.5016-03.2004

Conflict of Interest: The authors declare that the research was conducted in the absence of any commercial or financial relationships that could be construed as a potential conflict of interest.

Copyright (C) 2020 Xiao, Wang, Zhang, Zhou and Fu. This is an open-access article distributed under the terms of the Creative Commons Attribution License (CC BY). The use, distribution or reproduction in other forums is permitted, provided the original author(s) and the copyright owner(s) are credited and that the original publication in this journal is cited, in accordance with accepted academic practice. No use, distribution or reproduction is permitted which does not comply with these terms. 